\title{
Dynamic Mechanics of Rigid Helicopter Systems During Ditching
}

\author{
Mohamad Abu Ubaidah Amir Abu Zarima, Marja Azlima \\ Omar
}

Aircraft and helicopter often fly above open waters and thus have to observe regulations to ensure safe water landing under emergency conditions. This practice is also referred to as ditching - one of several types of slamming problems that are under review by the current regulations of the Federal Aviation Administration (FAA) and the European Aviation Safety Agency (EASA). Ditching is related to the controlled landing on water, with distinctive features such as hydrodynamic slamming loads, complex hydromechanics at tremendous forward speeds, as well as the interaction of multiphase fluid dynamics (air, water, and vapor). This paper presents the knowledge on system mechanics during helicopter ditching. The discussion begins with the fundamental kinetics of the rigid body, and then delves into dynamic relations to describe the effect of forces on motions. In the end, the paper discusses several relevant theories to further contribute to the understanding of the problem of impact.

\section{KEY WORDS}

$\sim$ Helicopter ditching

$\sim$ Water entry

$\sim$ Mechanics of ditching

a. Universiti Pertahanan Nasional Malaysia, Malaysia

e-mail: amir.a@upnm.edu.my

b. Universiti Malaysia Sabah, Malaysia

e-mail: mazlima5@gmail.com

doi: 10.7225/toms.v10.n02.013

This work is licensed under (cc) BY

Received on: 20.05.2021/Revised on: 28.07.2021/Accepted on: 01.08.2021/Published: 21.10.2021

\section{INTRODUCTION}

Aircraft and helicopters often fly above open waters and thus have to observe regulations that ensure safe water landing under emergency conditions, also referred to as ditching. This is particularly important for helicopters that are commonly used to support marine tasks, e.g. serve offshore platform. Ditching is related to controlled landing on water, and has some distinctive features, namely, hydrodynamic slamming loads, complex hydromechanics at tremendous forward speeds, and the interaction of multiphase fluid dynamics (air, water, and vapor).

The study of ditching dates back to Von Karman ( Von Kármán, 1929) and Wagner ( Wagner, 1932). Numerous studies have since considered a two-dimensional cross-section of a simple shape (wedge, cone, sphere, and cylinder) assuming that the structure is a rigid body. The water is generally modeled as an incompressible, irrotational, inviscid fluid. In early days, slamming during ship operation was the most explored design problem related to water impact.

Since slamming involves interaction between a structure and a free-surface fluid, research has expanded to other water entry problems such as water landing of solid rocket boosters and spacecraft, the ditching and water landing of aircraft, ballistic impacts on fuel tanks, and other applications (S. Abrate, 2013). This paper emphasized only principles relevant for further understanding of the current knowledge of helicopter ditching. Further in-depth review of hull slamming and water entry is provided in (Abrate, 2013). 


\section{BACKGROUND}

\subsection{Inertial Dynamics of Helicopters}

\subsubsection{Reference System}

The mock-up moves with six degrees of freedom (DOF). Thus, its motion is defined using three coordinates for translations and three coordinates for rotations. The axis systems considered are explained below (see Figure 1):

- $\quad$ Earth-fixed frame ( 0 -frame) $\left(\mathrm{O}, \mathrm{X}_{\text {ref }}, \mathrm{Y}_{\text {ref }}, \mathrm{Z}_{\text {ref }}\right)$ is a right-handed orthogonal reference frame fixed to the earth and considered Galilean. Positive $X_{\text {ref }}$ and $Y_{\text {ref }}$ are orthogonal in the horizontal plane and Zref is positive towards up. Origin $\mathrm{O}$ is located on calm water surface.

- Body-fixed frame for motion equations (b-frame) This reference frame is fixed to the body where $G$ is the Center of Gravity COG $\left(G, x_{b}, y_{b}, z_{b}\right)$. Positive $x b$ is pointed backwards from the nose, positive $y_{b}$ points starboard and positive $z_{b}$ points upwards. This frame is used to define the orientation of main particulars during free flight and impact. The translations in the study are monitored through the motions of $\mathrm{G}$ in the 0 -frame.

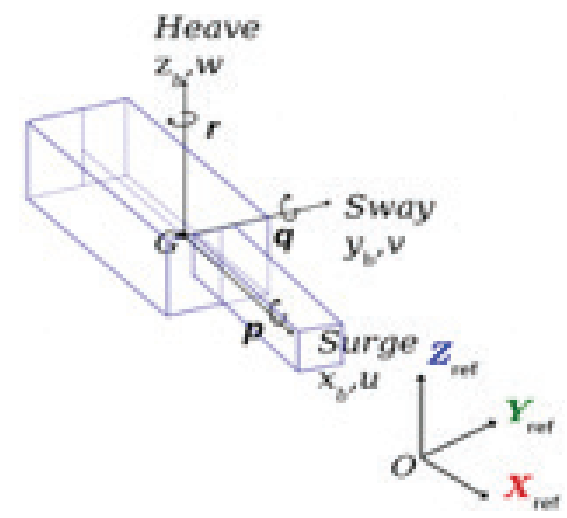

Figure 1.

Notation and sign convention description.

\subsubsection{Vector Notation}

The mathematical notation that allows the identification of position, velocity and acceleration points of interest for the mock-up had to be devised to express them in different frames, e.g. generic point of interest $p$ in the mock-up (in this explanation, $p$ and $f$ are only used to describe the sample notation, and are unrelated to any notation in Figure 1).

- $\quad r_{p}^{f}$ denotes the position of $p$ with respect to frame $f$ : $r_{p}^{f}=x_{p}^{f} f_{x}+y_{p}^{f} f_{y}+z_{p}^{f} f_{z}\left[\begin{array}{l}x_{p}^{f} \\ y_{p}^{f} \\ z_{p}^{f}\end{array}\right]=\left[x_{p}^{f}, y_{p}^{f}, z_{p}^{f}\right]^{T}$

- $\quad v_{p}{ }^{f}$ and $v_{p}{ }^{f}$ denote the velocity and acceleration of $p$ with respect to frame $f$.

- $\Theta_{\mathrm{ob}}$ is a vector of Euler angles that transport the 0-frame into the orientation of the $b$-frame.

- $\quad \omega_{o b}{ }^{b}$ denotes the relative angular velocity of the b-frame with respect to the 0 -frame, decomposed in the $b$-frame.

\subsubsection{Motion Coordinates and Reference Frames}

The earth-fixed position of the mock-up is defined by the original coordinates of the $b$-frame, $G$ relative to the 0 -frame:

$$
r_{G}{ }^{0}=\left[\begin{array}{c}
x_{G} \\
y_{G} \\
z_{G}
\end{array}\right]
$$

The mock-up attitude orientation is defined by the orientation of the $b$-frame relative to the 0 -frame, which is presented by three intrinsic rotations that take the 0 -frame into the b-frame defined roll $\Phi$, pitch $\theta$, and yaw $\Psi$. These rotations are called Tait-Bryan angles or Euler angles and are defined as:

$$
\Theta_{o b}=\left[\begin{array}{l}
\psi \\
\theta \\
\Phi
\end{array}\right]
$$

Therefore, the position orientation vector is defined as:

$\eta=\left[\begin{array}{c}R_{G}{ }^{0} \\ \Theta_{o b} b\end{array}\right]=\left[x_{G^{\prime}} y_{G}, z_{G}, \psi, \theta, \Phi\right]^{T}$

while the linear and angular velocity vector of the mock-up are conveniently expressed in the b-frame as:

$v=\left[\begin{array}{l}v_{G}^{b} \\ \omega_{o b}^{b}\end{array}\right]=[u, v, w, p, q, r]^{T}$

where $v_{G}{ }^{b}=[u, v, w]^{\top}$ is the linear velocity of point $G$ expressed in the $b$-frame, and $\omega_{o b}{ }^{b}=[p, q, r]^{\top}$ is the angular velocity of the $b$-frame with respect to the 0 -frame expressed in the b-frame. 
The velocity $v^{0}$ of any point of the solid in the galilean frame is expressed with $v^{0}=v_{G}{ }^{b}+\omega_{o b}{ }^{\circ} \times r^{b}$.

\subsubsection{Velocity transformations}

Vector coordinates between different frames can be transformed by using appropriate matrices. According to ( $T$. Perez, 2005), generic vector $t$ can be depicted either in frame 0 or in frame $b$ as:

$t=\left[\begin{array}{l}x_{t}^{0} \\ y_{t}^{0} \\ z_{t}^{0}\end{array}\right]^{0}=\left[\begin{array}{c}x_{t}^{b} \\ y_{t}^{b} \\ z_{t}^{b}\end{array}\right]^{b}$

This led to the transformation matrix with notation $R_{b}{ }^{0}\left(\Theta_{o b}\right)$ which can be expressed in the $b$-frame to the 0 -frame as:

$t^{0}=R_{b}^{0}\left(\Theta_{o b}\right) \cdot t^{b}$

where rotation matrix $R_{b}{ }^{0}\left(\Theta_{o b}\right)$ is obtained by three consecutive rotations around the principal axes:

$R_{b}^{0}\left(\Theta_{o b}\right)=R_{x, \Phi} R_{y, \theta} R_{z, \psi}$

where (T. Perez, 2005):

$$
\begin{aligned}
& R_{x, \Phi}=\left[\begin{array}{ccc}
1 & 0 & 0 \\
0 & c \Phi & -s \Phi \\
0 & s \Phi & c \Phi
\end{array}\right], \\
& R_{y, \theta}=\left[\begin{array}{ccc}
c \theta & 0 & s \theta \\
0 & 1 & 0 \\
-s \theta & 0 & c \Phi
\end{array}\right], \\
& R_{z, \psi}=\left[\begin{array}{ccc}
c \psi & -s \psi & 0 \\
s \psi & c \psi & 0 \\
0 & 0 & 1
\end{array}\right]
\end{aligned}
$$

$R_{b}{ }^{0}\left(\Theta_{o b}\right)=\left[\begin{array}{ccr}c \psi c \theta & -s \psi c \theta+c \psi s \theta s \Phi & s \psi s \Phi+c \psi c \theta s \Phi \\ s \psi c \theta & c \psi c \Phi+s \Phi s \theta s \psi & -c \psi s \Phi+s \Phi c \Phi s \theta \\ -s \theta & c \theta s \Phi & c \theta c \Phi\end{array}\right]$ where $\mathrm{c}=\mathrm{cos}, \mathrm{s}=\sin$ and $\mathrm{t}=\tan$. Thus:

$R_{b}^{0}\left(\Theta_{o b}\right)=R_{0}^{b}\left(\Theta_{o b}\right)^{-1} R_{b}^{0}\left(\Theta_{o b}\right)^{b}$

The transformation of velocities of the center of gravity is expressed in the $b$-frame $(u, v, w)$, while the time derivative of the position in the 0 -frame can be expressed as:

$\left[\begin{array}{c}\dot{x}_{G} \\ \dot{y}_{G} \\ z_{G}\end{array}\right]^{0}=R_{b}^{0}\left(\Theta_{o b}\right)=\left[\begin{array}{l}u \\ v \\ w\end{array}\right]$

Angular velocity vector $\omega_{o b}^{b}$ in the fixed-body frame, $b$-frame related to the time rate of change of Euler angles $\Theta_{o b}$ can be expressed as:

$\Theta_{o b}=T_{\Theta}\left(\Theta_{o b}\right) \cdot \omega_{o b}^{b} \quad$ or $\left[\begin{array}{c}\Phi \\ \Theta \\ \psi\end{array}\right]=\left(T_{\Theta} \Theta_{o b}\right)\left[\begin{array}{c}p \\ q \\ r\end{array}\right]$

where $T_{\Theta}\left(\Theta_{o b}\right)$ is the transformation matrix and its inverse given by:

$T_{\Theta}\left(\Theta_{o b}\right)=\left[\begin{array}{ccc}1 & s \theta t \theta & c \Phi t \theta \\ 0 & c \Phi & -s \Phi \\ 0 & s \Phi / c \theta & c \Phi / c \theta\end{array}\right]$

$T_{\Theta}\left(\Theta_{o b}\right)^{-1}=\left[\begin{array}{ccr}1 & s \theta t \theta & -s \theta \\ 0 & c \Phi & c \theta s \Phi \\ 0 & -s \theta & c \Phi / c \theta\end{array}\right]$

Transformation matrix $\mathrm{T}_{\Theta}\left(\Theta_{\mathrm{ob}}\right)$ can be derived from ( $\mathrm{T}$. Fossen, 2002):

$$
\begin{aligned}
\omega_{o b}^{b}=\left[\begin{array}{l}
p \\
q \\
r
\end{array}\right] & =\left[\begin{array}{l}
\Phi \\
0 \\
0
\end{array}\right]+R_{x, \Phi}{ }^{T}\left[\begin{array}{l}
0 \\
\theta \\
0
\end{array}\right]+R_{x, \Phi}{ }^{T} R_{y, \theta}^{T}\left[\begin{array}{l}
0 \\
0 \\
\psi
\end{array}\right] \\
& =T_{\Theta}\left(\Theta_{o b}\right)^{-1} \Theta_{o b}
\end{aligned}
$$




\subsection{Linear Momentum}

General definition of momentum $\mathrm{p}$ of the solid $\mathrm{R}$ is given as:

$p=\int_{R} v^{0} d m$

where $v^{0}$ is the velocity of elementary element $d m$ in the Galilean reference frame. Momentum at the center of gravity can be established as:

$p=\int_{R} v d m=\int_{R} \frac{d r^{0}}{d t} d m=\frac{d}{d t}\left(\int_{R} r^{0} d m\right)=\frac{d}{d t}\left(m r_{G}{ }^{0}\right)$

where

$$
\frac{d}{d t}\left(m r_{G}^{0}\right)=m v_{G}^{0}
$$

Notice that the time derivative in Equation (16) was placed outside the integral whose region of integration $R$ is timedependent. This intervention is justified because the center of gravity of a rigid body behaves as a material point, as explained in (O'Reilly, 2019; Casey, 1983).

\subsection{Angular Momentum and Moment of Inertia}

The angular momentum of a rigid body relative to its center of gravity and the fixed origin of the reference 0 -frame, 0 are denoted respectively as $h$ and $h_{0}$. By definition:

$h=\int_{R} r^{b} \times v^{0} d m, h_{0}=\int_{R} r^{0} \times v^{0} d m$

Expanding the angular momentum $\mathrm{h}$ :

$$
\begin{aligned}
h & =\int_{R} r^{b} \times\left(v_{G}^{0}+\omega_{o b}^{b} \times r^{b}\right) d m \\
& =\int_{R} r^{b} \times v_{G}^{0} d m+\int_{R} r^{b} \times\left(\omega_{o b}^{b} \times r^{b}\right) d m \\
& =\int_{R} r^{b} \times\left(\omega_{o b}^{b} \times r^{b}\right) d m
\end{aligned}
$$

Expanding the angular momentum $\mathrm{h}_{0}$ :

$h_{0}=\int_{R} r^{0} \times v^{0} d m=\int_{R}\left(r_{G}{ }^{0}+r^{b}\right) \times v^{0} d m=h+r_{G}{ }^{0} \times p$

Equation (19) can also be rewritten as:

$$
\begin{aligned}
h & =\int_{R} r^{b} \times\left(\omega_{o b}{ }^{b} \times r^{b}\right) d m \\
& =\int_{R}\left(\left(r^{b} \cdot r^{b}\right) \omega_{o b}{ }^{b}-\left(r^{b} \cdot \omega_{o b}^{b}\right) r^{b}\right) d m
\end{aligned}
$$

Using

$r_{b}=\left[\begin{array}{l}x_{b} \\ y_{b} \\ z_{b}\end{array}\right]^{o b}$

The expression can be developed into

$h=\omega_{0 b}^{b} \int_{R}\left(x_{b}^{2}+y_{b}{ }^{2}+z_{b}{ }^{2}\right) d m+\int_{R} r^{b}\left(x_{b} p+y_{b} q+z_{b} r\right) d m$

$=\int_{R}\left[\begin{array}{ccr}\left(y_{b}{ }^{2}+z_{b}{ }^{2}\right) & -x_{b} y_{b} & -x_{b} z_{b} \\ -x_{b} y_{b} & \left(x_{b}{ }^{2}+z_{b}{ }^{2}\right) & -y_{b} z_{b} \\ -x_{b} z_{b} & -y_{b} z_{b} & \left(x_{b}{ }^{2}+y_{b}{ }^{2}\right)\end{array}\right] \omega_{o b}^{b}$

where the matrix of inertia with respect to the center of gravity $\mathrm{I}_{\mathrm{g}}^{\mathrm{b}}$ appears:

$h=I_{g}^{b} \omega_{o b}^{b}$ 


\subsection{Newton/ Euler Second Law}

Mock-up motions in 3 degrees of freedom (DOF) were therefore computed by applying the standard Euler's law to the $b$-frame with the $G\left(G, x_{b}, y_{b}, z_{b}\right)$ coinciding with the COG. The inertia matrix remains constant when a reference frame is fixed to the body. However, the frame of reference now rotates with angular velocity $\omega_{0 b}{ }^{b}$, resulting in:

$\frac{d P}{d t}=m\left(\frac{d v_{G}^{b}}{d t}+\omega_{o b}^{b} \times v_{G}^{b}\right)=F_{b}$

$$
\frac{d h}{d t}=I_{g}^{b}\left(\frac{d \omega_{o b}^{b}}{d t}+\omega_{o b}^{b} \times I_{g}^{b} v_{G}^{b}\right)=M_{b}
$$

Where $m$ is mass, while $F_{b}=\left(F_{x}, F_{y}, F_{z}\right)$ and $M_{b}=\left(M_{x}, M_{y}\right.$ ,$\left.M_{z}\right)$ are the vectors of force and moment acting on the mockup. The vector of force, moment and translational velocity in the body-fixed reference b-frame can be transformed into their counterparts in the earth-fixed reference frame, 0-frame, as follows:

$$
\begin{aligned}
& F_{0}=R_{b}^{0}\left(\Theta_{0 b}\right) F_{b} \\
& M_{0}=R_{b}^{0}\left(\Theta_{0 b}\right) M_{b} \\
& v_{0}=R_{b}^{0}\left(\Theta_{0 b}\right) v_{G}^{b}
\end{aligned}
$$

\subsection{Energy Conservation}

The definition of the kinetic energy for the solid (mock-up) is given by:
$E_{k s}=\frac{1}{2} \int_{R} v^{2} d m$

when preceding definitions are applied, we get:

$E_{k s}=\frac{1}{2} m v_{G}^{b} \cdot v_{G}^{b}+\frac{1}{2} \omega_{o b}{ }^{b} \cdot l_{G}{ }^{b} \omega_{o b}{ }^{b}$

\subsection{Fluid Force on the Body}

The force exerted on the body by the fluid is given by the integral of the local stress T over the surface:

$E_{\text {fluid }}=\iint_{S} T d S$

This can also be expressed by using the Cauchy stress tensor:

$E_{\text {fluid }}=\iint_{S} \overline{\bar{\sigma}} n d S$

The Cauchy stress tensor can be decomposed in $\overline{\bar{\sigma}}=-p \overline{\bar{I}}+\overline{\bar{\tau}}$, with $p$ being the local pressure, $\overline{\bar{\tau}}$ the viscous stress tensor and $\mathrm{n}$ is a normal pointing outward of the body.

\section{SIMPLIFIED SOLUTIONS FOR WATER ENTRY OF RIGID BODIES}

Von Karman (Von Kármán, 1929) and Wagner (Mizoguchi and Tanizawa, 1996; Wagner, 1932) developed two simplified theories on the impact of rigid bodies on the free surface of a fluid. Both theories are widely used and have provided useful insight into impact dynamics. Their description of the issue of 2-dimensional water entry in calm waters is illustrated below. 


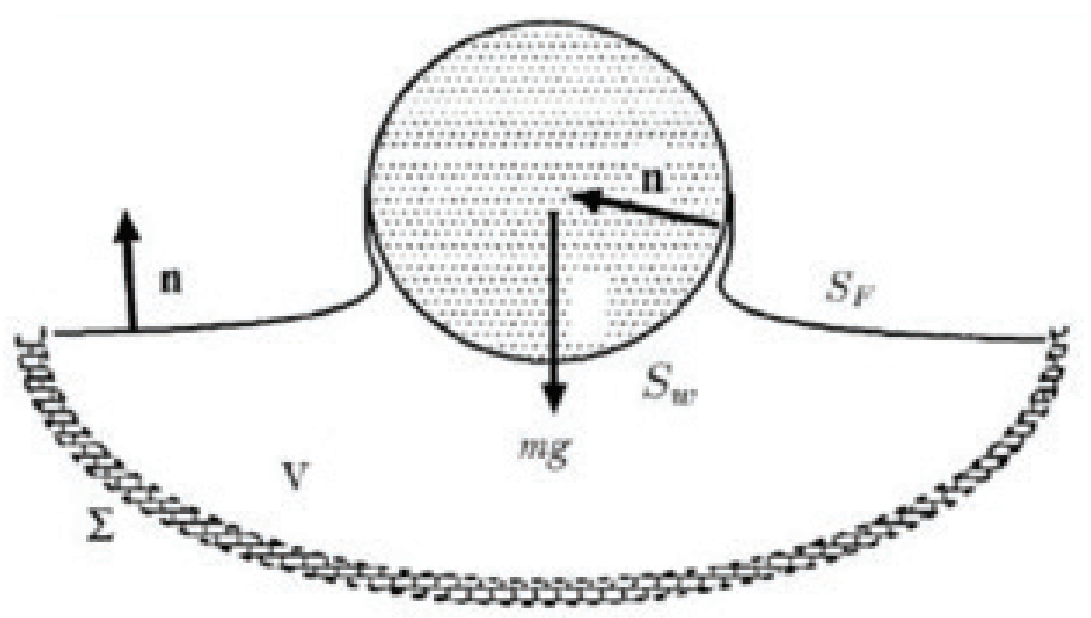

Figure 2.

Vertical impact.

This section examines body movement inside a fluid domain having volume $V$, limited by surface $S=S_{w} S_{F} \Sigma$, where $S_{w}$ is wetted body surface, $S_{F}$ is fluid surface, and $\Sigma$ is a patch in the water. The problem is illustrated in Figure 2.

\subsection{Von Karman's Approach}

When the Newton/Euler $2^{\text {nd }}$ law Equation (25) is applied to this problem, the external forces $F_{b}$ are the sum of the forces exerted by fluid $F_{\text {fluid }}$ and the weight $\mathrm{mg}$. To evaluate $\mathrm{F}_{\text {fluid }}$ using Equation (33) we need to know the stress across the body surface. Von Karman decomposed $F_{\text {fluid }}$ as the sum of the hydrodynamic impact force $F_{1}$, buoyancy force $F_{B}$, steady-state drag force $F_{D}$, and capillary force $F_{C}$. The motion equation can be written using Newton's second law as (S. Abrate, 2013):

$m v=m g-F_{1}-F_{B}-F_{C}-F_{D}$

$\mathrm{v}$ is velocity penetration relative to undisturbed water surface, where an overdot stands for the time derivative. During penetration, virtual mass $\mathrm{M}$ (added mass) of the fluid is considered to move with the body, so that the force applied by the fluid on the wetted surface of the body is equal and opposite to the force applied by the body on the fluid. The motion of the added mass is governed by:

$$
F=\frac{d}{d t} M v=M v+\frac{d M}{d \zeta} v^{2}
$$

If Equation (35) is substituted with Equation (34) the following expression is obtained:

$$
\frac{d}{d t}[(m+M) v]=m g-F_{B}-F_{c}-F_{D}
$$

With respect to the water entry problem, since the impact is rapid, water surface elevation, the effect of surface tension and the effect of viscosity and gravity on hydrodynamics of the fluid can be assumed to be very small and hence negligible. The motion in Equation (36) is then deduced as follows:

$$
\frac{d}{d t}(m+M) v=0
$$


Given that $\mathrm{t}=0$ is initial at impact, added mass $\mathrm{M}=0$, $\zeta^{*}(0)=v_{0^{\prime}}$ where $v_{0}$ is initial impact velocity. Integration with respect to time expresses the conservation of linear momentum throughout the water entry:

$$
m v_{0}=(m+M) v \text { or } \quad v=\frac{m v_{0}}{m+M}
$$

The development of Von Karman's approach is shown in the next subsection, in term of added mass. However, the impact load and added mass are likely to be underestimated, particularly for a small deadrise angle since this model neglects water surface elevation, as stated in (Mizoguchi and Tanizawa, 1996).

\subsubsection{In terms of added mass}

Impact transfers energy from the solid body to the fluid. When the integration from Newton's law is applied, the impulsemomentum principle can be determined as:

$\int_{t_{1}} t_{2} \frac{d}{d t}(M v) d t=\int_{t_{1}}{ }_{2} F d t_{i}(M v)_{2}-(M v)_{1}=\int_{t_{1}}{ }^{t_{2}} F d t$

where $M$ is added mass. The added mass is obtained from kinetic energy $E_{k f}$ of the fluid in motion (volume $V$ ), as follows:

$M=\frac{2 E_{k f}}{v^{2}}=\frac{\rho_{k f}}{v^{2}} \iiint_{V}(\nabla \Phi)^{2} d V$

When the last expression is transformed using Green's theorem and surface $S$ around volume $V$, the following expression is obtained:

$M=\frac{-\rho}{v^{2}} \int_{S} \Phi \frac{\partial \Phi}{\partial n} d S$

Given the complexity of each initial condition, defining the energy balance during impact is useful. The changes of kinetic energy exerted on the structure are as follows:

$$
\Delta E_{k s}=\frac{1}{2} m\left(v_{0}^{2}-v^{2}\right)=\frac{1}{2} m v_{0}^{2} \frac{m(2 m+M)}{\left(m+M^{2}\right)}
$$

while the changes of kinetic energy exerted on the fluid can be expressed as:

$\Delta E_{k f}=\frac{1}{2} M v^{2}=\frac{1}{2} m v_{0}^{2} \frac{m M}{(m+M)^{2}}$

At $\mathrm{t}=0$, added mass $\mathrm{M}=0$ and $\mathrm{v}_{0}$ is velocity at impact.

$\frac{\Delta E_{k f}}{\Delta E_{k s}}=\frac{m}{2 m+M}$

\subsection{Wagner's Approach}

Disregarding gravity and friction on the body surface, Wagner derived a more rational water impact theory that includes the hydrodynamic effect of small deadrise angles as discussed in (Abrate, 2013; Mizoguchi and Tanizawa, 1996). The hydrodynamic force is obtained from the pressure acting on the wetted surface $S_{w}$ of the body as follows:

$F_{\text {fluid }}=-\int_{S_{w}} p n d S$

where $\mathrm{p}$ is pressure and $\mathrm{n}$ is an outside normal of the body of the surface element dS. Fluid flow is governed by Navier-Stokes equations on the conservation of momentum and the continuity equations; both have an important connection with the fluid velocity field $v$.

During impact, flow can be considered irrotational and incompressible. First condition is that $(\nabla \times v=0)$ and velocity can be written in terms of potential function $\Phi$ :

$v=\nabla \varnothing$ 
With Incompressibility, the continuity equation becomes $\nabla \cdot v=0$. Hence Equation (45) can be rewritten as a Laplace's equation:

$\nabla \cdot v=\nabla \cdot(\nabla \Phi)=\nabla^{2} \Phi=0$

When Equation (45) is substituted with Navier-Stokes equations on the conservation of momentum, and the effects of viscosity and gravity are disregarded, Bernoulli's equation is obtained:

$$
\frac{P}{\rho}=\frac{\partial \Phi}{\partial t}-\frac{1}{2}(\nabla \Phi \cdot \nabla \Phi)
$$

which establishes a connection between pressure $p$ and velocity potential $\Phi$. However, identifying a potential function that meets both Laplace's and boundary conditions (should consist of wetted surface $S_{w}$ and free surface $S_{F}$, both of which are unknown) is problematic. The velocity of the body $v_{b}$ and the fluid are the same in the normal direction on the wetted surface, thus:

$v \cdot n=v_{b} \cdot n$

On the wetted surface, the velocity of the fluid in the normal direction can be expressed as $\frac{\partial \Phi}{\partial \mathrm{n}}=\mathrm{v} \cdot \mathrm{n}$. Given that the pressure
on the free surface $=0$ :

$$
\frac{\partial \Phi}{\partial n}+\frac{1}{2}(\nabla \Phi \cdot \nabla \Phi)=0
$$

Hence the force on the body in Equation (44) can be obtained using Bernoulli's equation:

$$
F_{\text {fluid }}=-\rho \int_{S_{w}}\left(\frac{\partial \Phi}{\partial n}+\frac{1}{2}(\nabla \Phi . \nabla \Phi)\right) n d S
$$

Wagner gave us a water impact theory that takes the hydrodynamic effect into account (S. Abrate, 2013; S. Mizoguchi and K. Tanizawa, 1996). Wagner's model is shown in Figure 3, where $c$ is the half width of the wet area, $\beta$ is the deadrise angle, and $v_{p}$ the velocity of the point of impact. In this model, also known as Wagner's expansion model, distance $c$ increases with the depth of penetration.

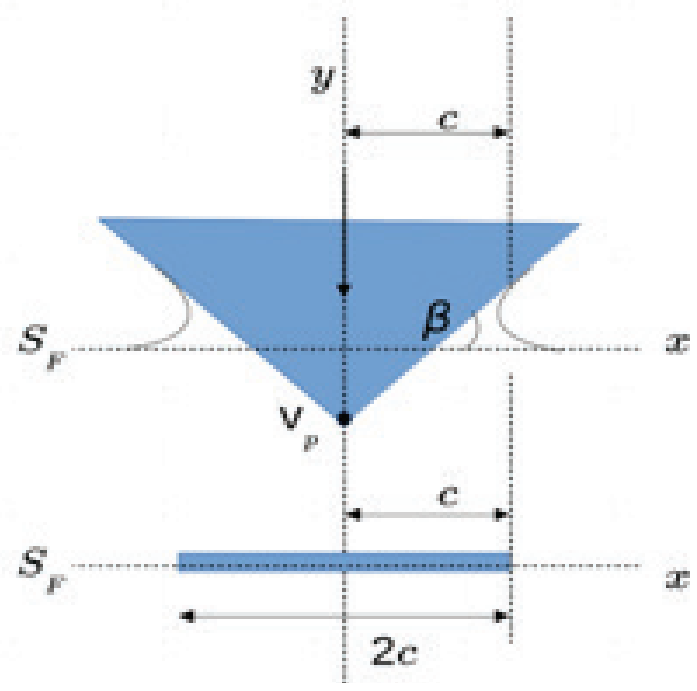

Figure 3.

Wedge entering the water (top) and Wagner's expansion model (bottom).

In two dimensions, the body is replaced with a flat plate having the length $2 \mathrm{c}$. The velocity vector is given as:

$v=\nabla \Phi=\frac{\partial \Phi}{\partial x} i+\frac{\partial \Phi}{\partial y} j$

While Wagner's potential is expressed as:

$\Phi=-v_{p} \sqrt{\left(c^{2}-z^{2}\right)} \quad$ where $z=x+i y$

The horizontal component of velocity along the plate given in $(|x|<c, y=0)$ is

$\frac{\partial \Phi}{\partial x}(x, 0)=v_{p} \frac{x}{\sqrt{\left(c^{2}-z^{2}\right)}}$ 
while the vertical component of velocity is zero along the bottom surface of the plate. Notice that the velocity of the fluid $\mathrm{v}$ is zero in the center $(\mathrm{x}=\mathrm{y}=0)$ and becomes infinite along the edge $(x=c, y=0)$. Hence, when Bernoulli's equation is applied in Equation (47), pressure along the bottom plate is obtained as follows:

$$
\frac{p(x)}{\rho}=\frac{\partial \Phi}{\partial x}\left(v_{p} \sqrt{\left.c^{2}-x^{2}\right)}-\frac{1}{2}\left(v_{p} \frac{x}{\sqrt{\left(c^{2}-x^{2}\right)}}\right)^{2}\right.
$$

Since the length of the bottom plate and velocity vary with time,

$$
\frac{p(x)}{\rho}=\sqrt{c^{2}-x^{2}} \frac{d v_{p}}{d t}+v_{p} \frac{c}{\sqrt{\left(c^{2}-x^{2}\right)}} \frac{d c}{d t}-\frac{1}{2} \frac{v_{p}^{2} x^{2}}{\sqrt{\left(c^{2}-x^{2}\right)}}
$$

Examine the equation on the right-hand side; given that $x \rightarrow c$, the first term tends to be zero, while the others become infinite. At the maximum penetration, when $v_{p}=v$ :

$$
c(t)=\left(\frac{\pi}{2}\right)\left(\frac{v(t)}{\tan \beta}\right) \text { hence } \frac{d c}{d t}=\frac{\pi v_{p} \cot \beta}{d t}
$$

If substituted and simplified, the following expression is obtained:

$$
p(x)=\frac{1}{2} \rho v_{p}^{2}\left[\frac{\pi \cot \beta}{\sqrt{1-x^{2} / c^{2}}}-\frac{x^{2} / c^{2}}{1-x^{2} / c^{2}}+\frac{2 v_{p}}{v_{p}^{2}} \sqrt{c^{2}-x^{2}}\right]
$$

where $c$ is the half width of the wet area, $\beta$ is the deadrise angle, and $v_{p}$ the velocity of the expanding flat plate in the uniform flow. Numerous studies have been conducted based on this theory, but only a few explored the issue in 3-dimensions, particularly in ship application, which is theoretically understood. Therefore, there is a need for 3-dimensional ditching theories and numerical methods.

\section{CONCLUSION}

This paper has presented fundamental equations required to calculate force during helicopter ditching events. Fixed and mobile reference systems for the issue of ditching have been introduced, together with mechanics equations useful for experiment analysis. Some information on water impact theories available have been given, while Von Karman's and Wagner's theories have been succinctly described. It was shown that impact load can be evaluated using the concept of added mass in either momentum or energy analysis without any contradiction. After impact, the body's mechanical energy is transferred to the fluid.

\section{ACKNOWLEDGEMENT}

Authors would like to thank Universiti Pertahanan Nasional Malaysia for grant UPNM/2021/GPJP/STG/1.

\section{REFERENCES}

Abrate, S., 2011. Hull Slamming. Applied Mechanics Reviews, 64(6). Available at: http://dx.doi.org/10.1115/1.4023571.

Casey, J., 1983. A Treatment of Rigid Body Dynamics. Journal of Applied Mechanics, 50(4a), pp.905-907. Available at: http://dx.doi.org/10.1115/1.3167171.

Fossen, T., 2002. Marine control systems: guidance, navigation and control of ships, rigs and underwater vehicles. Norwegian University of Science and Technology Trondheim, Norway.

Mizoguchi, S., and Tanizawa, K., 1996. Impact wave loads due to slamming. A Review Ship Technology Research, 43.

O’Reilly, O.M., 2019. Engineering Dynamics, 3rd Edition. Springer.

Payne, P.R., 1993. The spray sheets produced during vertical wedge impact and steady planing. Ocean Engineering, 20(3), pp.247-261. Available at: http://dx.doi.org/10.1016/0029-8018(93)90023-b.

Perez, T., 2005. Ship Motion Control: Course Keeping and Roll Stabilization Using Rudder and Fins. series Advances in Industrial Control. Springer.

Von Kármán, T., 1929. The impact on seaplane floats during landing. National Advisory Committee on Aeronautics, Washington, DC.

Wagner, H., 1932. Über Stoß- und Gleitvorgänge an der Oberfläche von Flüssigkeiten. ZAMM - Zeitschrift für Angewandte Mathematik und Mechanik, 12(4), pp.193-215. Available at:

http://dx.doi.org/10.1002/zamm.19320120402. 